\title{
Análisis factorial confirmatorio con mínimos cuadrados ordinarios no ponderados de una escala de actitudes hacia el duelo
}

\section{Confirmatory factor analysis ols unweighted a scale of attitudes toward the duel}

Julia del Carmen Chávez Carapia Javier Carreón Guillén

Jorge Hernández Valdés

Universidad Nacional Autónoma

de México

\author{
Cruz García Lirios \\ Universidad Autónoma del \\ Estado de México
}

\section{Resumen}

La teoría del morir señala que la tendencia demográfica de nacimientos, matrimonios, divorcios, esperanza de vida y defunciones evidencia un escenario de duelo permanente en la dinámica poblacional. En las entidades con una mayoría de habitantes jóvenes y, por consiguiente, una acelerada tasa de reproducción y natalidad como el Estado de México, existe una mayor probabilidad de duelo si se consideran los estilos de vida y conductas de riesgo. El objetivo del presente trabajo es confirmar mediante mínimos cuadrados la estructura factorial de la Escala de Actitudes hacia el Duelo (EAD) de García (2010) y discutir tanto sus alcances como límites frente a la Escala de Miedo a la Muerte (FDS), la Multiescala de Miedo a la Muerte (MFDS), la Escala de Depresión ante la Muerte (DDS) y la Escala de Propósito de Vida (PLS). Para tal propósito, se llevó a cabo un estudio no experimental y transversal con una selección no probabilística de 260 estudiantes de una universidad pública. El análisis permite advertir líneas de investigación que exploren la estructura multirasgo de la EAD.

Palabras clave: muerte, duelo, tanatología, actitud, miedo.

Nota del autor

Julia del Carmen Chávez Carapia, Departamento de Trabajo Social, Universidad Nacional Autónoma de México (UNAM)-Escuela Nacional de Trabajo Social (ENTS); Javier Carreón Guillén, Departamento de Trabajo Social, UNAM-ENTS; Jorge Hernández Valdés, Departamento de Trabajo Social, UNAMENTS; Cruz García Lirios, Departamento de Trabajo Social, Universidad Autónoma del Estado de México, Unidad Académica Profesional de Huehuetoca.

La correspondencia en relación con este artículo debe dirigirse a Cruz García Lirios, Departamento de Trabajo Social, Universidad Autónoma del Estado de México, Unidad Académica Profesional de Huehuetoca, Calle Nuestra Señora de los Ángeles S/N, Manzana 93, Colonia La Cañada, C.P. 54680, Municipio de Huehuetoca, Edo. de México, México.

Dirección electrónica: garcialirios@yahoo.com 


\begin{abstract}
Die theory states that the demographic trend of births, marriages, divorces, deaths and life expectancy evidence a scenario of permanent mourning in population dynamics. In the states with a majority of young people and therefore an accelerated rate of reproduction and birth as the State of Mexico, there is a greater probability of mourning when considering the lifestyles and risk behaviors. The aim of this work is confirmed by least squares the factorial structure of the scale of attitudes towards the Duel (EAD) Garcia (2010) and discuss both its scope and limits facing Scale Fear of Death (FDS), the multiscale Fear of Death (MFDS), Depression Scale to Death (DDS) and Life Purpose Scale (PLS). a non-experimental and cross-sectional study with a nonrandom selection of 260 students of a public university was held. Research on the structure of ODL multitrait will explore are noticed.
\end{abstract}

Keywords: death, mourning, thanatology, attitude, factorial analysis.

Con base en la teoría del bien morir, se definió el objetivo del presente estudio: confirmar la estructura factorial de la EAD para discutir sus alcances y límites frente a la FDS, la MFDS, la DDS y la PLS.

La teoría del bien morir afirma que el duelo y las actitudes son aspectos consustanciales a la muerte. Desde la psicología de la salud, la atención tanatológica en casos de enfermedad terminal o fallecimientos inminentes, consiste en el análisis de las disposiciones de los grupos ante el fenómeno. Sin embargo, en términos poblacionales y demográficos, los fallecimientos ocupan un tercer sitio con respecto a los nacimientos, los matrimonios y los divorcios (ver figura 1). Durante el periodo que va de 2004 a 2011, los nacimientos superaron a los fallecimientos, matrimonios y divorcios.

La prevalencia de los nacimientos no sólo está vinculada con los matrimonios, se han incrementado en mayor medida que los demás rubros poblacionales debido a la tendencia que impulsa la actitud a favor de la vida; aunque la planificación familiar sea una asignatura pendiente de la política demográfica, la sociedad mexicana parece no estar preparada ante el duelo que supone la muerte.

De este modo, la esperanza de vida en el periodo 2010 a 2012 se mantuvo constante, por lo tanto, se evidenciaron las diferencias entre hombres (70 años de esperanza de vida) y mujeres (78 años de esperanza de vida). En promedio (74 años de esperanza de vida), la población mexicana parece contar con una estructura de cuidado, matrimonios más que divorcios y mujeres con mayor esperanza de vida que los hombres, características favorables para la preservación de la vida, pero vulnerables ante la muerte (ver figura 2). 


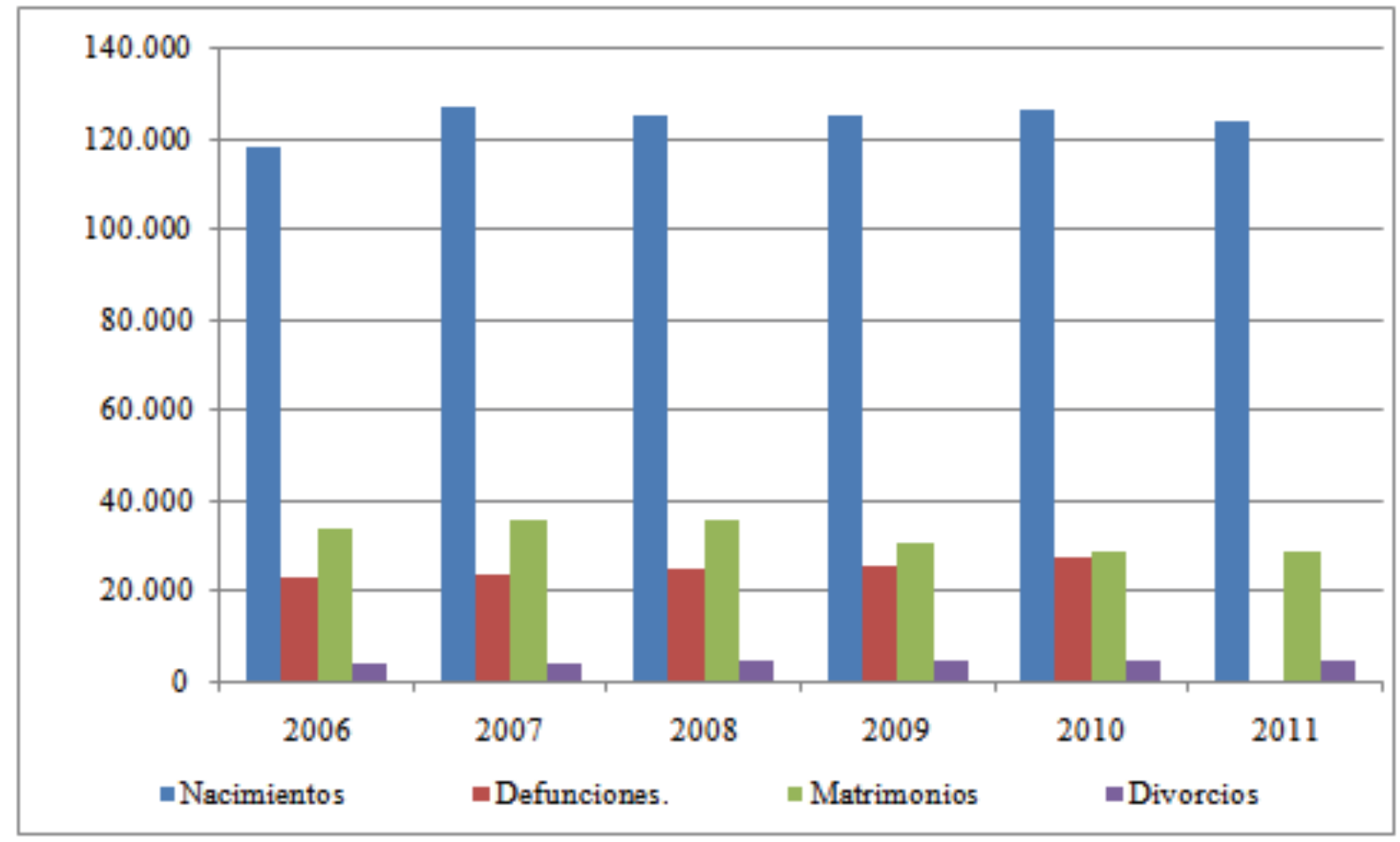

Figura 1. Variables poblacionales. Fuente: INEGI (2015).

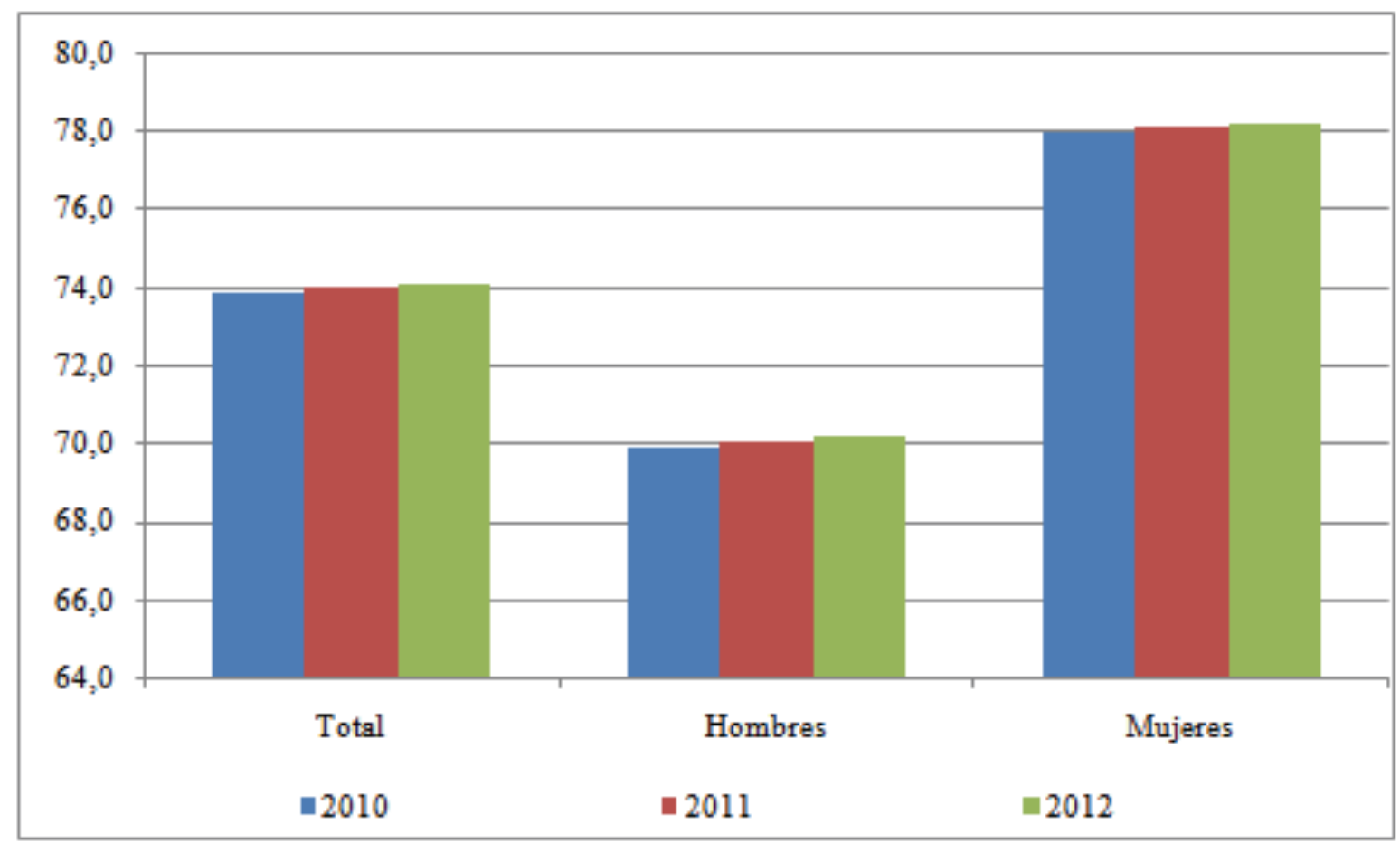

Figura 2. Esperanza de vida. Fuente: INEGI (2015). 
A pesar de los registros anteriores, la vulnerabilidad ante la muerte y el duelo que supone ésta tienen características particulares, ya que si bien la mayoría de las defunciones corresponde al grupo de 65 y más años, así como al grupo de entre 45 y 64 años, las muertes en menores de un año son mayores que las del resto de los grupos divididos en rangos de edad (ver figura 3).

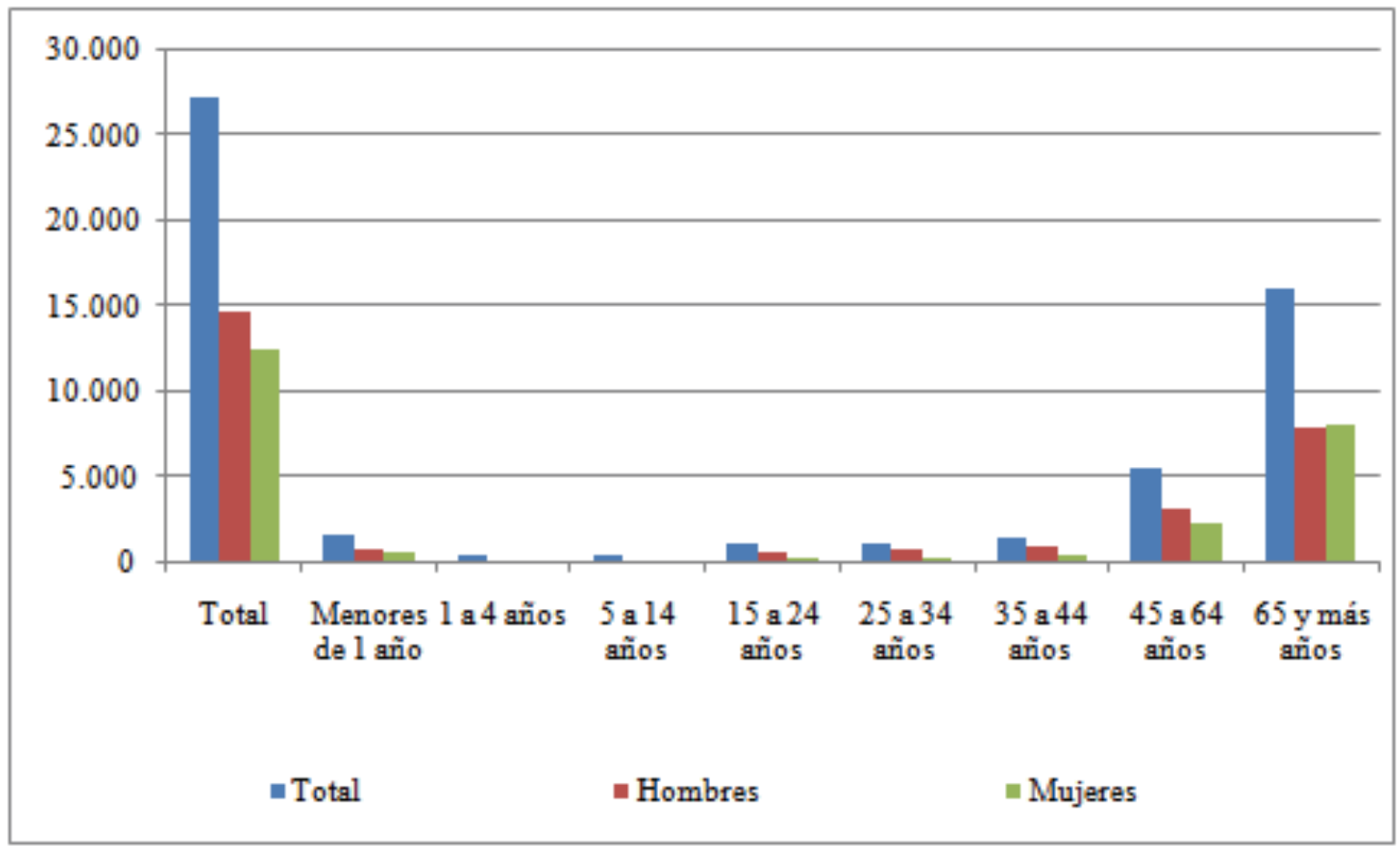

Figura 3. Defunciones en 2010. Fuente: INEGI (2015).

Aunada a la tendencia de nacimientos y la esperanza de vida que suponen una actitud a favor de la existencia, aunque se presenta la vulnerabilidad ante la pérdida de un ser cercano o querido, el sistema de salud pública no cuenta con un proceso terapéutico específico para cada caso (Quintanar \& García, 2010). En el ámbito de la medición de las actitudes hacia la muerte, el miedo en diferentes dimensiones, según los contextos y las muestras de estudio, ha ganado presencia.

Espinosa, Sanhueza y Barriga (2011) demostraron las propiedades psicométricas de la FDS con base cuatro dimensiones relativas: 1) miedo a la propia muerte, 2) miedo al proceso de morir propio, 3) miedo a la muerte de otros y 4) miedo al proceso de morir de otros. Los autores encontraron una consistencia interna de 
la escala general y de las subescalas superior al mínimo requerido (alfas mayores a 0.70 ); sin embargo, determinaron cinco dimensiones alusivas a: 1) miedo a la muerte de otros, 2) miedo a la muerte propia, 3) miedo al proceso de morir físico, 4) miedo psicológico al morir, 5) miedo al proceso de morir de otros. Cada uno de los factores explicó $60 \%$ de la varianza total. No obstante, cinco dimensiones establecidas, la validez de la FDS en un contexto diferente y con muestras distintas corrobora los supuestos teóricos con respecto a los hallazgos empíricos.

Villa y Suárez (2015) establecieron la validez de la FDS, a partir del hallazgo de cuatro factores alusivos a: 1) miedo a la propia muerte, 2) miedo al proceso de morir propio, 3) miedo a la muerte de otros y 4) miedo al proceso de morir de otros de la segunda escala. Por consiguiente, los investigadores dedujeron que individuos habituados a los sistemas autoritarios tienen más miedo a la muerte que los ciudadanos formados en sistemas democráticos. Esto es así porque las creencias en torno a la seguridad son inherentes a las actitudes hacia la muerte. Una mayor incidencia del Estado en el duelo colectivo está asociada a un creciente miedo a morir.

La medición de la FDS se ha vinculado con el sentido de la existencia. Young et al., (2015) encontraron que la FDS se correlacionó negativamente con la PLS y positivamente con la DDS. Cada una de estas escalas alcanzó una consistencia interna superior a la mínima requerida (alfa mayor a 0.70). Aunque dos de las tres escalas efectivamente miden actitudes y emociones negativas de la muerte y la vida, sólo la escala del propósito de la vida pondera un umbral que va de la pérdida de sentido de existencia hasta un pleno sentido de vida. Por consiguiente, la medición de la muerte, aun y cuando está delimitada al miedo, no se circunscribe a la probabilidad de duelo que las variables demográficas y poblacionales advierten según la tendencia global y local.

La medición del miedo a la muerte puede implicar un servicio de orientación tanatológica, pero delimitado a una fase transitoria del ser humano más que a una condición prevaleciente ante los riesgos que supone la dinámica poblacional. Power y Smith (2008) llevaron a cabo un estudio que incluyó las variables sociodemográficas. Para tal fin, utilizaron la MFDS y establecieron la confiabilidad (alfas superiores a 0.70 ) y la validez (pesos factoriales superiores a 0.300 ) de ocho dimensiones; 1) el miedo al proceso de morir, 2) el miedo a la muerte, 3) miedo a ser destruido, 4) miedo a la muerte de otros, 5) miedo a lo desconocido, 6) miedo a la conciencia de morir, 7) miedo a lo posterior de la muerte, 8) miedo al morir prematuramente. En tal sentido, observaron que las mujeres y los creyentes religiosos tenían más miedo a la muerte con respecto a hombres y no creyentes, quienes por su parte tenían más miedo a lo posterior de la muerte.

La virtud de la MFDS consiste en el establecimiento de diferencias entre grupos de personas con respecto a ocho dimensiones del miedo a la muerte. Si la estructura factorial del 
miedo a la muerte incluye ocho dimensiones, entonces el análisis factorial confirmatorio explicaría las diferencias entre la teoría del miedo a la muerte y las soluciones factorías ponderadas a partir de múltiples rasgos. Tal multiplicidad de factores de la MFDS delimitaría la atención al duelo. Barreto, De la Torre y Pérez (2012) establecieron una relación estrecha entre los instrumentos y las mediciones del duelo con respecto al apoyo emocional de pacientes oncológicos. Los modelos de atención centrados en el duelo más que en los síntomas muestran un avance en la concepción de la vida en relación con la muerte, pero los alcances y los límites de este modelo están en los deudos, ya que la diversidad de significados determinaría un modelo integral y más humano. En situaciones extremas tales como los eventos terroristas, la muerte y el duelo son temas centrales en la agenda pública, aunque en las políticas de seguridad prevalece más bien la violencia del Estado contra quienes considera sus enemigos (Gómez, 2007).

En tal contexto, las instituciones, programas y estrategias de seguridad contemplan la atención a víctimas sólo desde la indemnización económica, sin considerar los procesos de duelo. Incluso los sistemas e instituciones de salud sólo toman en cuenta la profesionalización de los cuidados paliativos ante la muerte inminente; para los profesionales de la salud, los fallecimientos en casa son el escenario ideal para la atención del duelo (Ascencio, Allende \& Verastegui, 2014). En el caso de la muerte súbita o de situaciones extremas de fallecimiento o pérdida de alguna extremidad, el estrés postraumático ha sido propuesto como una fase del duelo.

Crespo y Gómez (2012) establecieron la confiabilidad y la validez de un instrumento que midió los síntomas y el acontecimiento traumático. En relación con otros instrumentos -Escala de Gravedad de Síntomas, Escala Diagnóstica de Estrés Postraumático, Cuestionario de Eventos Perturbadores-, la Escala de Evaluación Global del Estrés Postraumático (EGEP) obtuvo correlaciones altas y significativas. La relación existente entre el miedo a la muerte y el servicio de atención postraumática se reviste así de sentido, ya que ambos procesos están conectados mediante el despliegue de emociones.

García (2010) demostró la configuración de tres factores de primer orden (afectivo, racional e intencional), así como la de uno de segundo orden actitudinal general. Tales dimensiones se estructuraron a partir de tres grupos de cinco reactivos, que incluían el mismo contenido, pero que se diferenciaban en las opciones de repuesta. Es decir, en torno al objeto actitudinal (atención tanatológica), los deudos tienden a estructurar sus sentimientos, razones e intenciones directa y positivamente. Sin embargo, la actitud en relación con la muerte cercana o inminente, no siempre es parte de un proceso racional, deliberado y sistemático, tal es el caso de las disposiciones ante infectados de VIH. Carreón et al. (2015) determinaron una relación directa $\mathrm{y}$ significativa entre las normas familiares $\mathrm{y}$ 
gremiales con respecto a las actitudes de los profesionales de la salud. Las actitudes hacia portadores de una enfermedad que se considera está relacionada con una muerte incierta o próxima está en función del soporte familiar de apoyo y las redes sociales a las que el infectado pertenece o quiere pertenecer (García et al., 2013). García et al. (2014) señalan que las actitudes de los profesionistas de la salud con respecto a enfermos terminales o pacientes que mueren intempestivamente en los centros de salud y hospitales públicos son determinados por su compromiso laboral, que es un sello distintivo de las organizaciones de la salud pública.

En efecto, la cultura laboral ha sido considerada como un determinante de las actitudes de los profesionales de la salud con respecto a la vida o muerte de sus pacientes. Carreón et al. (2013) advierten una relación estrecha entre el clima de relaciones y el de tareas, donde las actitudes indican un compromiso significativo cuando el clima de relaciones prevalece sobre las funciones, pero en una situación inversa, cuando los objetivos y las metas son prioritarios, la calidad de la atención se reduce a su mínima expresión.

En síntesis, los estudios psicológicos de la salud muestran que la muerte, el duelo y la atención tanatológica son aspectos consustanciales a las actitudes que se generan en los profesionistas de la salud, enfáticamente en aquellas profesiones que dedican un alto porcentaje de sus funciones al contacto directo con individuos en fase terminal, grupos cercanos a ellos, tales como familiares o amigos, así como con otros profesionistas que observan el fallecimiento de los infectados de VIH, enfermos de cáncer, adultos mayores demenciados, casos de muerte prematura o víctimas del terrorismo o la delincuencia organizada. En este sentido, los modelos de atención a víctimas y casos terminales parecen ajustarse a políticas de salud, o bien, a esquemas culturales y organizacionales internos de los centros y hospitales de salud pública.

Incluso, la literatura revisada advierte de los límites de los modelos de orientación al bien morir, aunque en lo concerniente a las actitudes es posible advertir tres ventajas con respecto a las variables medidas por el estado del conocimiento; 1) en tanto disposiciones, evaluaciones y opiniones, las actitudes son repositorios de aspectos sociales, institucionales, familiares y personales que; 2) están relacionados con la toma de decisiones y el desempeño de los profesionistas de salud, definiendo; 3) la calidad de la atención y el servicio de acompañamiento, bien morir o superación personal del trauma o duelo que supone la pérdida de un ser querido, o el estrés que implica el padecimiento de una enfermedad terminal, así como la impronta de un fallecimiento o mutilación de una extremidad.

El servicio de atención postraumática con respecto a las actitudes hacia la muerte ha transitado por tres fases.

La primera aproximación al estudio del duelo ante la muerte supuso seis fases: 1) negación, 2) 
aislamiento, 3) enojo, 4) regateo, 5) depresión y 6) aceptación, pero en los casos de muerte culposa, las fases resultaron insuficientes al no considerar los efectos de la culpa individual y colectiva en los deudos, por consiguiente, emerge un círculo de violencia simbólica.

El segundo enfoque consideró: 1) negación, 2) confusión, 3) enojo, 4) culpa, 5) soledad, 6) alivio y 7) restablecimiento. Tal propuesta resultó innovadora al incluir la atención de la culpa, la soledad y el restablecimiento, pero soslayó aquellos casos de muerte súbita o repentina, así como el shock simbólico en los deudos.

Un tercer modelo surgió e incluyó: 1) shock, 2) búsqueda, 3) desesperanza, 4) reorganización.
A partir de la hipótesis del equilibrio energético, el modelo plantea un equilibrio entre las actitudes a favor de la vida y a favor de la muerte. A diferencia del primer enfoque funcional, en el cual la vida prevalece como una condición esencial del ser humano conformista, o de la segunda aproximación normativa, en donde el duelo es una fase transitoria de los grupos, la tercera propuesta alude la importancia de entender la coexistencia de la vida con la muerte en términos colectivos. O lo que es lo mismo, la muerte como la vida no se reducen a un duelo personal o grupal, sino colectivo o social, incluso generacional, histórico o civilizatorio.

La especificación del modelo (ver figura 4) incluye tres premisas hipotéticas derivadas de la teoría del bien morir (ver figura 4).

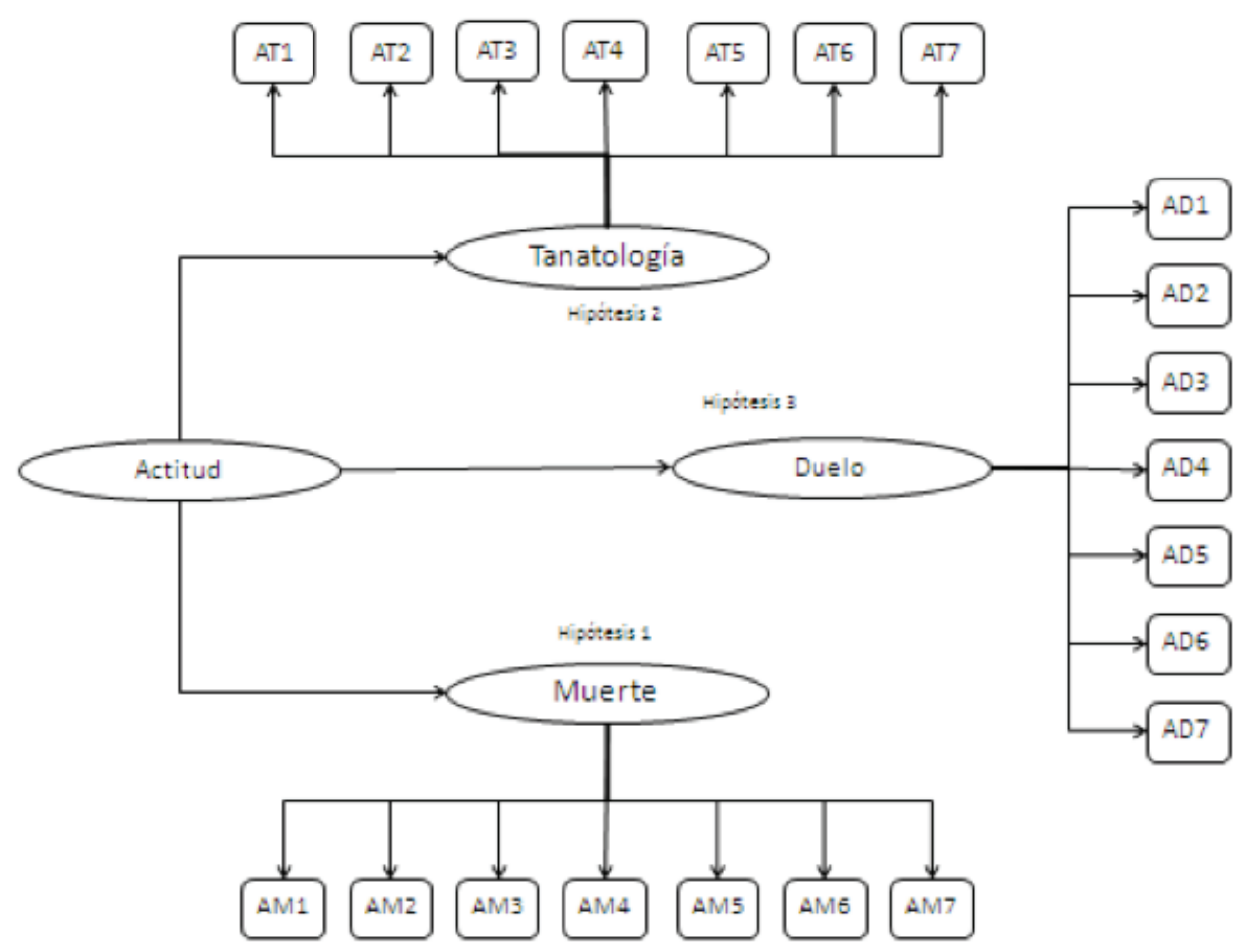

Figura 4. Especificación del modelo. Fuente: García (2010). 


\section{Premisa 1 (actitudes hacia la muerte).} La relación entre los eventos traumáticos con respecto a la actitud desfavorable a la muerte exacerban los síntomas de un duelo prolongado que minimizaría cualquier terapia de bien morir o aceptación de una pérdida.

Premisa 2 (actitud hacia el duelo). El proceso que requiere un evento traumático, como la muerte o la pérdida de una extremidad propia o de una persona cercana, está vinculado con las disposiciones que heredamos del grupo al que pertenecemos y de las actitudes que aprendemos del grupo al que queremos pertenecer.

Premisa 3 (actitud hacia la atención tanatológica). Las dimensiones de las actitudes hacia las terapias de apoyo son resultado de la diversidad de eventos traumáticos, pero también son producto del sesgo de disposiciones hacia la vida sin cuestionamiento de su fragilidad o la posibilidad de perderla.

\section{Método}

\section{Diseño}

Se llevó a cabo un estudio no experimental, trasversal y exploratorio.

\section{Formulación}

¿La EAD, con respecto a FDS, MFDS, DDS y PLS, mide tres dimensiones que permitirían el diagnóstico, la atención y evaluación del duelo en centros de salud pública con población vulnerable?

\section{Hipótesis nula}

La EAD incluye tres dimensiones -muerte, duelo y tanatología-, que se ajustan a los datos observados.

\section{Hipótesis alterna}

Las tres dimensiones de la EAD son diferentes a la estructura factorial ponderada.

\section{Variables}

Actitud hacia la muerte. Refiere a disposiciones heredadas y aprendidas con respecto a la prevalencia de estilos de vida, más que estilos de muerte o estilos de pérdida de una extremidad o persona cercana.

Actitud hacia el duelo. Consiste en la tendencia a ignorar o minimizar la pérdida de la vida, una persona cercana o la mutilación de una extremidad con respecto a otros estímulos vitales.

Actitud hacia la atención tanatológica. Es un conjunto de disposiciones desfavorables al apoyo emocional o terapéutico con respecto a la pérdida de la vida, un ser cercano o la mutilación de una extremidad.

\section{Muestra}

Se realizó una selección no probabilística de 260 estudiantes de una universidad pública del Estado de México (centro de México). Dicha muestra fue elegida intencionalmente en virtud de que la formación del servicio de atención postraumática es un requerimiento de los centros de salud pública (escenario de prácticas profesionales, servicio social y 
ejercicio profesional). De tal sección, 56\% lo representaron las mujeres y el resto, los hombres. Del total de estudiantes, $44 \%$ declaró tener menos de 18 años $(M=17.24$ y $D E=0.82)$, $20 \%$ señaló que su edad oscilaba entre 18 y 22 años $(M=21.24$ y $D E=0.73)$ y $36 \%$ restante dijo que su edad era mayor a los 22 años $(M=$ 24.35 y $D E=2.35)$. Asimismo, $35 \%$ recalcó que sus ingresos familiares mensuales eran menores a 3,500 pesos $(M=3,024$ y $D E=14.25), 40 \%$ ubicó sus ingresos entre los 3,500 y los 7,000 pesos mensuales $(M=4,362$ y $D E=243.24)$ y $25 \%$ restante mencionó un ingreso superior a 7,000 pesos al mes $(M=8,392$ y $D E=345.43)$.

\section{Instrumentos}

Se utilizó la Escala de Actitudes hacia la Muerte, el Duelo y la Tanatalogía (EAMDT) de García et al. (2013). El instrumento consta de 21 reactivos con cinco opciones de respuesta que van desde $0=$ nada de acuerdo, hasta $4=$ muy de acuerdo.

\section{Procedimiento}

Se empleó la técnica Delphi para homogeneizar los significados de "muerte", "duelo" y "tanatología". Además, se piloteó el instrumento en una muestra intencional de deudos. El instrumento final fue aplicado en las instalaciones de la universidad pública a la que pertenecen los estudiantes, previa garantía de anonimato y protección de datos personales. La información fue procesada en el Paquete
Estadístico para Ciencias Sociales (SPSS por sus siglas en inglés), versión 21 y Lisrel versión 8.8 .

\section{Análisis de datos}

La validez del instrumento se estableció con un análisis factorial confirmatorio de mínimos cuadrados ordinarios.

\section{Análisis Factorial Exploratorio (AFE).}

El AFE supone que: 1) se desconocen las dimensiones de un instrumento y éstas se establecen a partir de los resultados empíricos, 2) los factores dependen de la comunalidad (cantidad de varianza explicada que una combinación de los ítems ejerce sobre un ítem en particular) entre los ítems, 3) cada ítem ajusta su medición al factor que supuestamente pertenece al tener una mayor correlación -peso factorial-, 4) por consiguiente, la varianza del ítem está incluida en la varianza que tanto el ítem como el factor comparten, 5) los valores estandarizados se refieren a la saturación de los ítems en el factor y al error de medida; es la suma de todas las saturaciones elevada al cuadrado evidencia la cantidad de varianza explicada - eigenvalue de cada factor-. Por lo tanto, 6) todos los factores correlacionan -rotación oblicua-, o ningún factor está asociado -rotación ortogonal-.

Debido a que la rotación varimax refiere a un criterio ortogonal entre una matriz de estructura en relación con una matriz de configuración, donde se asume que ambas son similares, ésta 
se recomienda cuando no hay un factor preponderante, ya que se asume la independencia de los factores. Incluso, el desarrollo de los software ha permitido un criterio universal (ortogonal y/u oblicuo), aunque la tendencia es utilizar criterios oblicuos, la rotación ortogonal alcanzó un $80 \%$ de los estudios revisados frente Al 12\% de las estructuras claras, simples e interpretables desde el criterio oblicuo.

Por lo tanto, un criterio ortogonal supone un alejamiento de la realidad si los factores del constructo no son independientes, reflejarán altas correlaciones frente a correlaciones bajas -menores a 0.30 - desde un criterio oblicuo, entonces es recomendable un criterio ortogonal.

\section{Análisis Factorial Confirmatorio (AFC).}

El AFC parte de supuestos tales como: 1) establecer a priori la estructura de los datos mediante el contraste de un modelo teórico, que orienta las relaciones de dependencia entre factores de primer y segundo orden con respecto a indicadores, 2) establecer a posteriori las correlaciones o covariazas, explicándolas con variables observadas y latentes, 3) considerar que la puntuación de cada respondiente ante un ítem es generada por un factor latente, el cual explica la variabilidad de los indicadores, 4) empero, el factor latente no explica toda la variabilidad de los ítems -error de medida-, 5) por consiguiente, separa la varianza de cada indicador y extrae la varianza explicada por el factor y el error de medida; separa ambas mediciones y calcula sus coeficientes por separado, es decir, sólo trabaja con la varianza representativa del indicador con respecto al factor y controla las fuentes de variación. Debido a lo anterior, 6) las correlaciones entre los factores pueden establecerse o no a priori, aunque 7) los índices de verosimilitud, ajuste y residual pueden recomendar una especificación del modelo.

Respecto al error de medida, puede deberse a que 1) los indicadores proceden de un mismo concepto, 2) sesgos en las opciones de respuesta, y 3 ) redacción de ítems con un contenido teórico múltiple.

\section{Técnica de ajuste racional: Mínimos}

\section{Cuadrados Ordinarios no Ponderados.}

La estimación de factores se realizó con Mínimos Cuadrados Ordinarios no Ponderados, lo cual supone: 1) una distribución indeterminada de las variables categóricas, organizadas en matrices policóricas; 2) la solución factorial debe ser determinada con residuales cercanos a cero tanto como sea posible, ya que la factorización de ítems ordinarios permitió el análisis e interpretación de la matriz de correlaciones policóricas; 3) se minimiza la suma de los cuadrados de las diferencias entre las matrices de correlaciones observadas y las matrices de correlaciones reproducidas, es más recomendable con respecto a otros protocolos; 4) la aplicación del método funciona mejor que otras cuando la muestra es pequeña, las variables son diversas y el número de factores a establecer es pequeño; 5) se evita la aparición 
de casos Heywood (saturaciones mayores que la unidad y varianza de error negativas), frecuentes en otros métodos de estimación.

\section{Resultados}

La escala general obtuvo una confiabilidad suficiente $($ alfa $=0.792)$, la subescala de Actitud hacia la Muerte $($ alfa $=0.782)$, subescala de Actitud hacia el Duelo (alfa $=0.770)$ y la subescala de Actitud hacia la Tanatología (alfa $=0.791)$. Lo anterior implica que la consistencia interna del instrumento permite observar datos similares en contextos y muestras diferentes (ver tabla 1).

Tabla 1

Descriptivos, confiabilidad y validez del instrumento

\begin{tabular}{|c|c|c|c|c|c|c|c|}
\hline & Ítem & M & $\mathrm{DE}$ & Alfa & $\mathrm{F} 1$ & $\mathrm{~F} 2$ & F3 \\
\hline & Subescala de Actitud hacia la muerte & & & 0,782 & & & \\
\hline AM1 & Venimos a esta vida para gozar placenteramente de ella & 1,02 & 0,20 & 0,781 & 0,493 & & \\
\hline AM2 & El bien morir es para la gente anciana & 1,43 & 0,36 & 0,704 & 0,471 & & \\
\hline AM3 & Vivir la vida es buscar la felicidad & 1,56 & 0,37 & 0,721 & 0,311 & & \\
\hline AM4 & La muerte es para quien ignora los placeres de la vida & 1,24 & 0,21 & 0,793 & 0,682 & & \\
\hline AM5 & Cada quien busca la felicidad de la existencia antes de que se agote & 1,46 & 0,44 & 0,706 & 0,345 & & \\
\hline AM6 & Quienes están imposibilitados para vivir admiran a quienes son felices & 1,76 & 0,82 & 0,758 & 0,568 & & \\
\hline \multirow[t]{2}{*}{ AM7 } & La forma de morir es una consecuencia de cómo se vivió la vida & 1,47 & 0,41 & 0,736 & 0,320 & & \\
\hline & Subescala de Actitud hacia el Duelo & & & 0,770 & & & \\
\hline AD1 & El estrés de perder a alguien es permanente & 3,05 & 0,21 & 0,724 & & 0,583 & \\
\hline AD2 & Mi vida gira en torno al bienestar de las personas cercanas & 3,54 & 0,43 & 0,735 & & 0,605 & \\
\hline AD3 & Tantas pérdidas me hicieron superarlas rápidamente & 3,67 & 0,54 & 0,714 & & 0,371 & \\
\hline AD4 & Llorar por alguien que se ha ido es un reacción del momento & 3,58 & 0,56 & 0,717 & & 0,546 & \\
\hline AD5 & Vestir de negro es respetar el luto de quienes conocieron al fallecido/a & 3,42 & 0,55 & 0,791 & & 0,423 & \\
\hline AD6 & Las pérdidas son parte de la vida & 3,01 & 0,67 & 0,732 & & 0,581 & \\
\hline \multirow[t]{2}{*}{ AD7 } & La muerte es una extensión de la vida & 3,94 & 0,52 & 0,785 & & 0,430 & \\
\hline & Subescala de Actitud hacia la Tanatología & & & 0,791 & & & \\
\hline AT1 & A menudo, los apoyos emocionales sólo empeoran la pérdida & 2,56 & 0,42 & 0,731 & & & 0,446 \\
\hline AT2 & Es mejor olvidar que recordar terapéuticamente a quienes se han ido & 2,58 & 0,46 & 0,706 & & & 0,613 \\
\hline AT3 & $\begin{array}{l}\text { Hasta el día de hoy la tanatología sólo ayuda a quienes esperaban una } \\
\text { pérdida }\end{array}$ & 2,31 & 0,57 & 0,743 & & & 0,691 \\
\hline AT4 & La muerte súbita es incomprensible con o sin terapia & 2,40 & 0,68 & 0,744 & & & 0,506 \\
\hline AT5 & La tanatología es un discurso de quienes se aprovechan de los deudos & 2,85 & 0,82 & 0,721 & & & 0,481 \\
\hline AT6 & El luto es proceso exclusivo de quienes conocieron al fallecido/a & 2,37 & 0,31 & 0,720 & & & 0,302 \\
\hline AT7 & El bien morir es algo que podemos aprender sin apoyo emocional & 2,07 & 0,47 & 0,743 & & & 0,551 \\
\hline
\end{tabular}

Nota: Técnica de Ajuste Racional: Mínimos Cuadrados Ordinarios no Ponderados; Boostrap = 0.000; KMO=0.671; prueba de Bartlett $[\chi 2=14.24(9 \mathrm{gl}) p=0.000] \mathrm{F} 1=$ Actitud hacia la Muerte (35\% de la varianza total explicada), F2 = Actitud hacia el Duelo $(14 \%$ de la varianza total explicada), F3 = Actitud hacia la Tanatología ( $8 \%$ de la varianza total explicada). Fuente: Elaborada con los datos del presente estudio. 
Cada uno de los factores se estableció con pesos factoriales superiores a 0.300 previa adecuación y esfericidad [Bootstrap $=0.000$; $K M O=0.671 ; \chi 2=14.24(9 g l) p=0.000]$. La validez se estimó con el peso factorial y el porcentaje de la varianza explicada. En el caso del primer factor relativo a la Actitud hacia la Muerte explicó 35\% de la varianza total, el factor de la Actitud hacia el Duelo explicó 4\% de la varianza y el factor de la Actitud hacia la Tanatología explicó $8 \%$ de la varianza (ver figura 5).

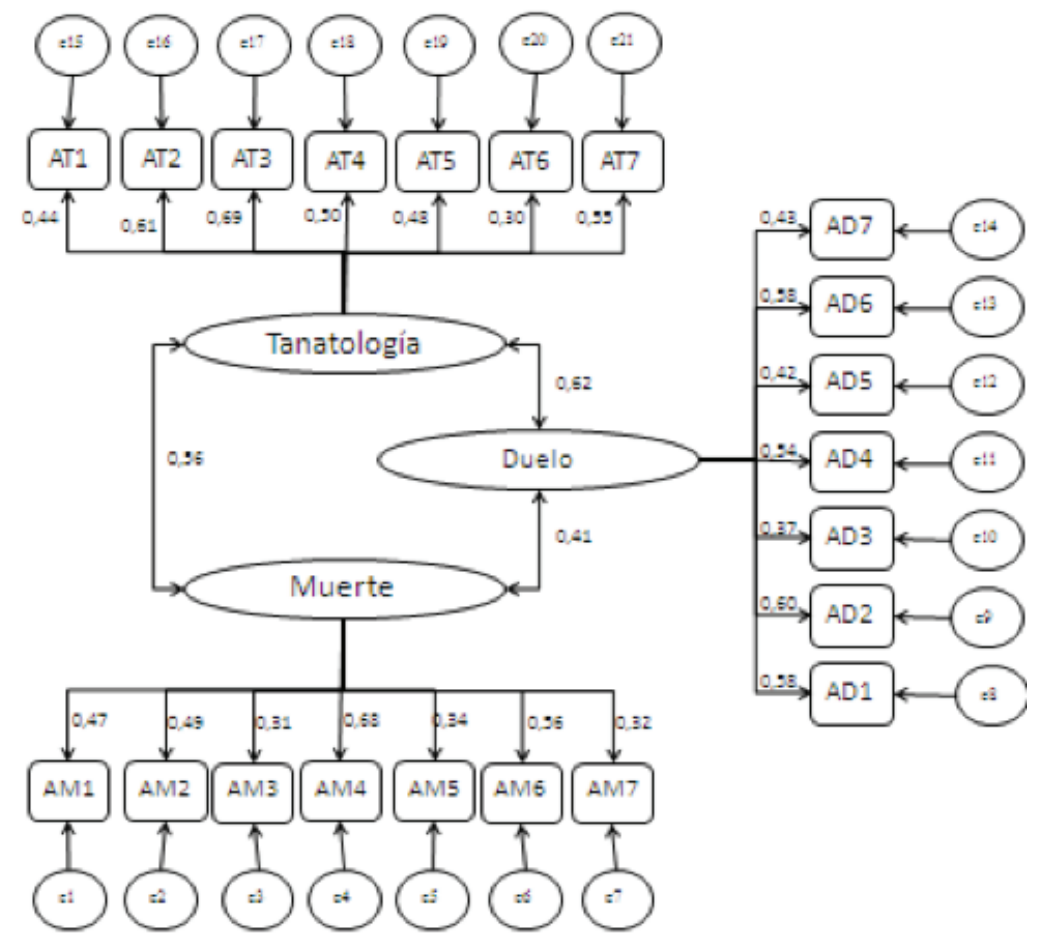

Figura 5. Modelo estructural. Fuente: Elaborada con los datos del estudio.

Los parámetros de ajuste y residual $\lceil\chi 2=$ $243.35(32 \mathrm{gl}) \mathrm{p}=0.034 ; G F I=0.995 ; C F I$ $=0.997 ;$ RMSEA $=0.003 \mathrm{~J}$, hipótesis nula de ajuste entre el modelo especificado y el modelo ponderado fue aceptada.

\section{Discusión}

El aporte del presente estudio al estado del conocimiento estriba en la confiabilidad (suficiente entre 0.70 y 0.79 ) y la validez establecida con mínimos cuadrados ordinarios 
no ponderados de la EAD en una muestra no probabilística de estudiantes de Ciencias de la Salud y el Comportamiento en una entidad con elevada tasa de reproducción sexual y natalidad.

En relación con otros instrumentos, como la FDS, la MFDS, la DDS y la PLS, la EAD mide un proceso continuo de disposiciones ante la muerte que no se agota ahí y pondera sus efectos en el duelo y la atención postraumática. En el ámbito de la salud pública, la medición de la actitud hacia la muerte, el duelo y el servicio terapéutico -tanatología-, es indispensable para evaluar las competencias requeridas por los centros de salud para con sus prestadores de servicio social, practicantes profesionales o profesionistas residentes. Precisamente, es en este contexto donde la EAD tiene una ventaja comparativa con respecto a los otros instrumentos, ya que permite la evaluación del desempeño de los profesionistas de la salud.

En tal sentido, futuras líneas de investigación en torno a la formación de profesionistas de la salud, especializados en la atención postraumática, serviría para anticipar escenarios de calidad de los servicios de salud relacionados con enfermedades terminales, muertes accidentales, conductas de alto riesgo, defunciones y duelos. No obstante que la validez del EAD se puede establecer con un criterio oblicuo y un método de ejes principales, se recomienda emplear un criterio ortogonal y un método de mínimos cuadrados ordinarios no ponderados debido a las ventajas descritas en el apartado de análisis de datos.

\section{Referencias}

Ascencio, L., Allende, S., \& Verastegui, E. (2014). Creencias, actitudes y ansiedad ante la muerte en un equipo multidisciplinario de cuidados paliativos oncológicos. Psiconcología, 11(1), 101-115. doi: 10.5209/rev_ PSIC.2014.v11.n1.44920

Barreto, P., De la Torre, O., \& Pérez, M. (2012). Detección del duelo complicado. Psiconcología, 9(2), 355-368. doi: 10.5209/rev_ PSIC.2013.v9.n2-3.40902

Carreón, J., Bustos, J., Hernández, J., Quintero, M., \& García, C. (2015). Confiabilidad y validez de un instrumento que mide actitud hacia grupos cercanos a portadores de VIH/ SIDA. Eureka, 12(2), 218-230. ISSN: 22180559.

Carreón, J., García, C., Morales, M., \& Rosas, F. (2013). Compromiso laboral en el trabajo social del sector salud. Invurnus, 8(2), 15-23. Crespo, M., \& Gómez, M. (2012). La evaluación del estrés postraumático: Presentación de la Escala de Evaluación Global del Estrés Postraumático (EGEP). Clínica y Salud, 23(1), 25-42. doi: 10.5093/c12012a4

Espinosa, M., Sanhueza, O., \& Barriga, O. (2011). Validación de la Escala del Miedo a la Muerte de Collete-Lester en una muestra de estudiantes de enfermería. Revista Latinoamericana de Enfermería, 19(5), 1-10.

García, C., Carreón, J., Hernández, J., \& Morales, M. (2014). Contraste de un modelo de compromiso laboral en centros de salud pública. Acta Universitaria, 24(1), 48-59. 
García, C. (2010). La estructura de la actitud hacia la atención tanatológica. Fundamentos en Humanidades, 11(1), 121-132.

García, C., Carreón, J., Hernández, J., \& Bustos, J. (2013). Actitud de trabajadoras sociales hacia portadores del Virus de Inmunodeficiencia Humana en centros de salud comunitaria. Salud \& Sociedad, 4(1), 60-68. ISSN: 0718-7475.

Gómez, J. (2007). La muerte y el acompañamiento del morir. International Journal of Development and Educational Psichology, 2, 115-132. ISSN: 0114-9877.

Power, T., \& Smith, S. (2008). Predictors of fear of death and self-mortality: An Atlantic Canadian perspective. Death Studies, 32, 253272. doi: 10.1080/07481180701880935

Quintanar, F., \& García, C. (2010). Intervención tanatológica para población anciana: Hacia una psicotanatología basada en la evidencia. Journal of Behavior Health \& Social Ussues, 2(2), 11-23. doi: 10.5460/jbhsi.v2.2.26785

Villa, M., \& Suárez, C. (2015). Autoritarismo y conciencia de muerte en estudiantes universitarios españoles. Revista de Psicología de la Universidad de Chile, 24(2), 1-21. doi: 10.5354/0719-0581.2015.38402

Young, A., Ju, H., So, K., \& Hye, C. (2015). Attitude toward death and the purpose in life among Korean adolescents. Advanced Science and Technology Letters, 16(1), 204208. doi: 10.14257/astl.2015.116.41
Recibido el 12 de noviembre de 2015 Revisado el 10 de febrero de 2016 Aceptado el 25 de abril de 2016 